\title{
Heavy Higgs boson production and decay into top quarks at the LHC
}

\author{
Werner Bernreuther ${ }^{a}$, Peter Galler ${ }^{* b}$, Clemens Mellein ${ }^{a}$, Zong-Guo Si ${ }^{c}$ and Peter Uwer ${ }^{b}$ \\ ${ }^{a}$ Institut für Theoretische Teilchenphysik und Kosmologie, \\ RWTH Aachen University, 52056 Aachen, Germany \\ ${ }^{b}$ Institut für Physik, Humboldt-Universität zu Berlin, 12489 Berlin, Germany \\ ${ }^{c}$ School of Physics, Shandong University, Jinan, Shandong 250100, China \\ E-mail: breuther@physik.rwth-aachen.de, galler@physik.hu-berlin.de, \\ mellein@physik.rwth-aachen.de, zgsi@sdu.edu.cn, uwer@physik.hu-berlin.de
}

\begin{abstract}
In this contribution we report on the calculation of the next-to-leading order (NLO) QCD corrections to the hadro-production of heavy neutral Higgs bosons and their decay into top-quark pairs within the type-II two-Higgs-doublet extension of the standard model (SM). We take into account the contributions from resonant Higgs boson production, the non-resonant SM $t \bar{t}$ background as well as the interference of these two contributions. The NLO corrections to the signal and interference contributions are calculated by applying the heavy top-quark mass $\left(m_{t}\right)$ limit including an effective rescaling. In our NLO calculation the QCD-Higgs interference is evaluated in the resonance region that provides the dominant part of the heavy Higgs-boson contributions. Evaluating representative $C P$-conserving and $C P$-violating parameter scenarios within the twoHiggs-doublet model (2HDM) we present results for different distributions and, in addition, for observables that depend on the top-quark spin.
\end{abstract}

38th International Conference on High Energy Physics

3-10 August 2016

Chicago, USA

${ }^{*}$ Speaker. 


\section{Introduction}

Now that the standard-model like Higgs boson has been experimentally $[1,2]$ established at the LHC, the ongoing and future investigation of the scalar sector-including the search for additional spin-zero bosons-remains one of the central research goals at the LHC. In this work, we investigate additional heavy Higgs bosons $\phi$ with masses $m_{\phi}>2 m_{t}$ and unsuppressed couplings to top quarks. Thus these heavy Higgs bosons can appear as resonances in the $t \bar{t}$ decay channel. So far dedicated searches by ATLAS [3, 4] and CMS [5, 6] do not show any excess above the SM $t \bar{t}$ continuum. However, until now the experimental analysis suffered from the limited statistics of Run I and the fact that only leading-order (LO) predictions for this process were available. Only very recently QCD NLO corrections have been calculated [7, 8].

Here we report on the results of the computation of NLO QCD corrections to heavy Higgs boson production and decay to top quarks presenting inclusive as well as differential cross sections at a center of mass energy $\sqrt{s}=13 \mathrm{TeV}$ at the LHC. In addition, we study $t \bar{t}$ spin correlations. Our NLO calculation takes the following contributions into account: the resonant production of heavy Higgs bosons and their decay into $t \bar{t}$, the SM non-resonant contribution to $t \bar{t}$ production and the interference of these two processes. We apply the heavy top-quark mass limit to the calculation of the NLO QCD corrections while at LO we keep the full $m_{t}$ dependence. An additional rescaling is used to improve the prediction of this approximation. The calculation of the QCD-Higgs interference is restricted to the resonant region which gives the dominant heavy Higgs-boson contribution. Details of the calculation as well as analytical results are presented in [7]. Our approach can be applied to a broader spectrum of new physics models featuring heavy spin-zero bosons that couple to $t \bar{t}$. However, we choose a specific model to account for the decay width of the heavy Higgs bosons in a consistent fashion because the effects of heavy Higgs bosons on $t \bar{t}$ production depend on the bosons' decay width. As a specific model, we chose the UV-complete type-II 2HDM with no tree-level flavor-changing neutral currents.

\section{Two-Higgs-doublet model scenarios}

We study the type-II 2HDM within the so-called alignment limit, i.e. the lightest of the three neutral Higgs bosons is identified with the observed $125 \mathrm{GeV}$ Higgs boson with SM-like couplings. Furthermore, we set the masses $m_{2,3}$ of the other two heavy neutral Higgs bosons to $m_{2,3}>2 m_{t}$. In the next section, we present the results of four type-II 2HDM parameter scenarios where the Higgs bosons have the same or slightly enhanced top-quark Yukawa coupling strengths compared to the SM strength $m_{t} / v$. Scenarios 1,2 and 4 are $C P$-conserving while scenario 3 is $C P$-violating. While in scenario 1 the two heavy Higgs bosons have similar masses, scenarios 2-4 feature wellseparated Higgs boson masses. A summary of all input parameters as well as the Yukawa couplings and decay widths for the four scenarios is shown in Tab. 1. For the definition of the parameters and further details on scenarios 1-3 we refer to [7]. Details of the 2HDM in general and type-II in particular can be found in the literature, e.g. [9].

\section{Results}

In this section we present results for the NLO QCD corrections to heavy Higgs production and decay to $t \bar{t}$ within the type-II $2 \mathrm{HDM}$ using the two approximations (heavy-top limit and restriction to resonance region) mentioned in the introduction. 


\begin{tabular}{|c|c|c|c|c|}
\hline & scenario 1 & scenario 2 & scenario 3 & scenario 4 \\
\hline \multicolumn{5}{|c|}{ input parameters } \\
\hline $\tan \beta$ & 0.7 & 0.7 & 0.7 & 1 \\
\hline$v[\mathrm{GeV}]$ & 246 & 246 & 246 & 246 \\
\hline$m_{+}[\mathrm{GeV}]$ & $>720$ & $>720$ & $>720$ & $>720$ \\
\hline$m_{1}[\mathrm{GeV}]$ & 125 & 125 & 125 & 125 \\
\hline$m_{2}[\mathrm{GeV}]$ & 550 & 550 & 500 & 400 \\
\hline$m_{3}[\mathrm{GeV}]$ & 510 & 700 & 800 & 900 \\
\hline$\alpha_{1}$ & $\beta$ & $\beta$ & $\beta$ & $\beta$ \\
\hline$\alpha_{2}$ & 0 & 0 & $\pi / 15$ & 0 \\
\hline$\alpha_{3}$ & 0 & 0 & $\pi / 4$ & 0 \\
\hline \multicolumn{5}{|c|}{ calculated parameters } \\
\hline$\Gamma_{2}[\mathrm{GeV}]$ & 34.56 & 34.49 & 36.55 & 3.99 \\
\hline$\Gamma_{3}[\mathrm{GeV}]$ & 49.28 & 75.28 & 128.16 & 162.39 \\
\hline$a_{t 1}$ & 1 & 1 & 0.978 & 1 \\
\hline$b_{t 1}$ & 0 & 0 & 0.297 & 0 \\
\hline$a_{t 2}$ & 1.429 & 1.429 & 0.863 & 1 \\
\hline$b_{t 2}$ & 0 & 0 & 0.988 & 0 \\
\hline$a_{t 3}$ & 0 & 0 & -1.157 & 0 \\
\hline$b_{t 3}$ & 1.429 & 1.429 & 0.988 & 1 \\
\hline
\end{tabular}

Table 1: $2 \mathrm{HDM}$ parameter settings for scenarios 1-4. The SM-like Higgs boson $\phi_{1}$ has a decay width $\Gamma_{1} \approx 4 \mathrm{MeV}$. It plays no role in our analysis.

Spin independent observables: We calculated, for scenarios $1-3$, a number of observables that do not depend on the top-quark spin. The inclusive $t \bar{t}$ cross section is shown in Tab. 2. The central value of the renormalization and factorization scales is denoted by $\mu_{0}=\left(m_{2}+m_{3}\right) / 4$. The scales are changed simultaneously by a factor of 2 and $1 / 2$, respectively. The resulting change in the total cross section is indicated in this table by the superscripts and subscripts. $\sigma_{\mathrm{QCDW}}$ is the $t \bar{t}$ cross section due to QCD and weak interaction at NLO and $\sigma_{2 \mathrm{HDM}}$ denotes the contributions from resonant heavy Higgs production and decay to $t \bar{t}$ and the interference with the non-resonant SM $t \bar{t}$ production at NLO QCD. Table 2 shows that the ratio of the $2 \mathrm{HDM}$ and the SM contribution is only about $1-2 \%$. A higher sensitivity can be achieved by studying differential cross sections. In Fig. 1 we present results for the top quark pair invariant mass $M_{t \bar{t}}$ distribution for scenarios $1-3$ at NLO QCD. In the upper plots, the SM contribution and the SM+2HDM contribution including the interference is shown in black and red, respectively. The blue shaded area indicates the uncertainty due to renormalization and factorization scale variations. In the lower plots, the signal-to-background ratio at LO (NLO) is displayed in green (red). The lower-lying Higgs resonance leads to a peak-dip structure in the $M_{t \bar{t}}$ distribution that is caused by the interference of signal and background. In the inclusive $t \bar{t}$ cross section the peak and dip partly cancel. As a result the effects of the additional Higgs bosons are significantly reduced as the numbers of Tab. 2 show. For avoiding this cancellation we applied cuts on $M_{t \bar{t}}$ to restrict the observables to a regime below and above the resonance. This is indicated by the hatched region in the ratio plots in Fig. 1. We investigated, with these $M_{t \bar{t}}$ bins, the differential cross section with respect to the top-quark rapidity, the top-quark transverse momentum and the cosine of the Collins-Soper angle $\cos \theta_{\mathrm{CS}}$. Among these observables the Collins-Soper angle is the most sensitive one. We show the result in Fig. 1 only for scenario 1 where the effects are largest due to the overlapping resonances. The maximum signal-to-background (S/B) ratios in the $M_{t \bar{t}}$ distribution and Collins-Soper angle distribution in the lower $M_{t \bar{t}}$ bin are of similar size ( $\gtrsim 6 \%$ ). But in the case of the $M_{t \bar{t}}$ distribution one needs a small bin size to reach this sensitivity. Considering the inclusive cross section in the 
range $390 \mathrm{GeV} \leq \mathrm{M}_{\overline{t t}} \leq 540 \mathrm{GeV}$ one obtains a reduced value for $\mathrm{S} / \mathrm{B}$ of $\sim 4 \%$. This also shows that measurements of the $M_{t \bar{t}}$ distribution with a large bin size tend to wash out the effects of additional heavy Higgs bosons in $t \bar{t}$ production.

\begin{tabular}{llll}
\hline \hline & Scenario 1 & Scenario 2 & Scenario 3 \\
\hline$\mu_{0}[\mathrm{GeV}]$ & 265 & 312.5 & 325 \\
$\sigma_{\mathrm{QCDW}}[\mathrm{pb}]$ & $643.22_{-77.71}^{+81.23}$ & $624.25_{-76.19}^{+80.98}$ & $619.56_{-75.72}^{+81.05}$ \\
$\sigma_{2 \mathrm{HDM}}[\mathrm{pb}]$ & $13.59_{-1.64}^{+1.85}$ & $7.4_{-0.78}^{+0.77}$ & $7.21_{-0.77}^{+0.81}$ \\
$\sigma_{2 \mathrm{HDM}} / \sigma_{\mathrm{QCDW}}[\%]$ & 2.1 & 1.2 & 1.2 \\
\hline \hline
\end{tabular}

Table 2: Inclusive $t \bar{t}$ cross sections at NLO QCD with and without the heavy Higgs resonances.
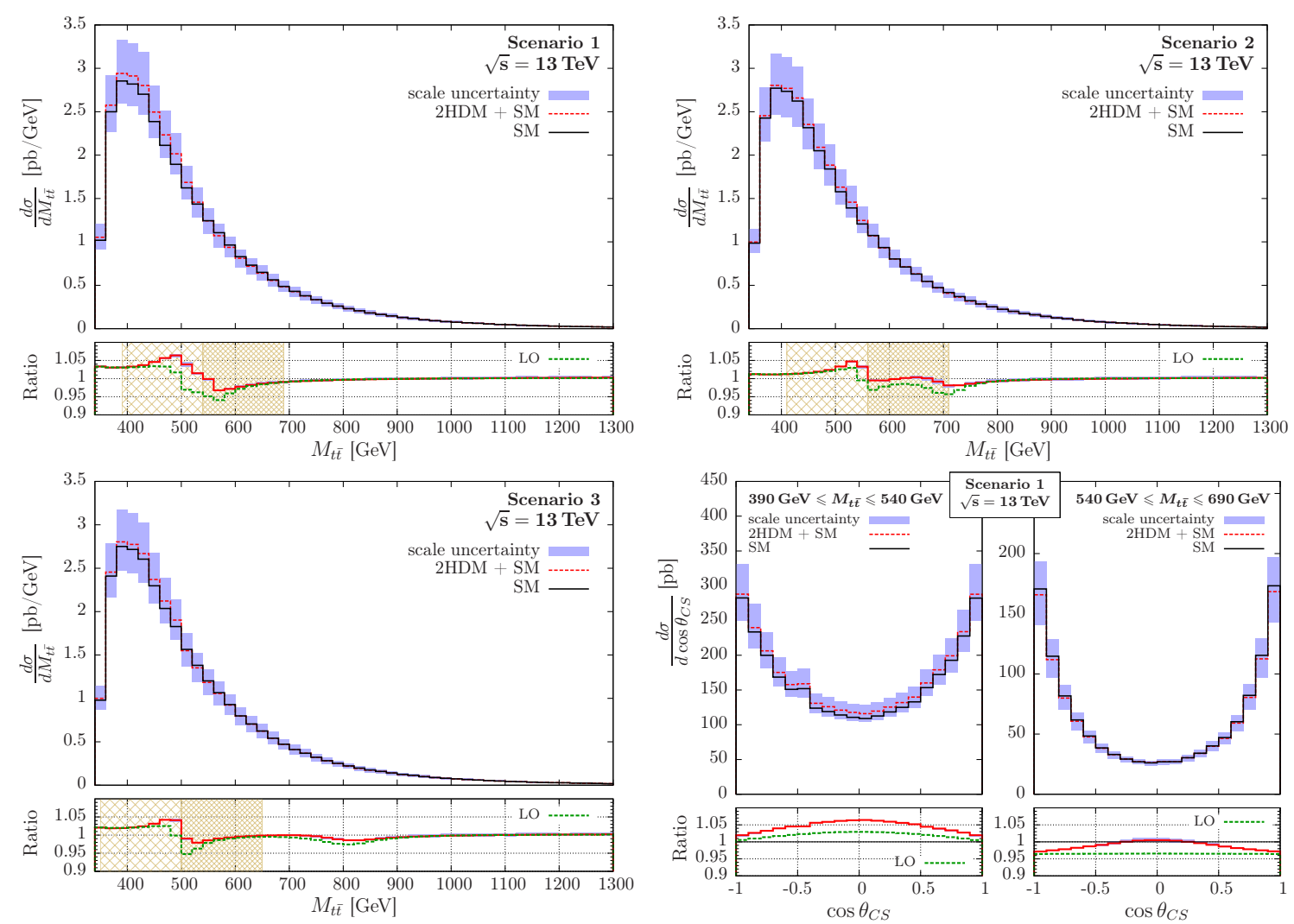

Figure 1: Upper left: $M_{t \bar{t}}$ distribution for scenario 1, upper right: $M_{t \bar{t}}$ distribution for scenario 2 , lower left: $M_{t \bar{t}}$ distribution for scenario 3, lower right: Collins-Soper angle distribution for scenario 1 in two different $M_{t \bar{t}}$ bins.

Spin correlations: We investigated also the polarization of the top quark and $t \bar{t}$ spin correlations for several type-II $2 \mathrm{HDM}$ scenarios. Here, we present only the results for $t \bar{t}$ spin correlations in scenario 4 and refer for a detailed account of spin dependent observables to a future publication [10]. We calculated four spin correlation observables $C_{\mathrm{kk}} \equiv C_{\mathrm{hel}}, C_{\mathrm{nn}}, C_{\mathrm{rr}}$ and $D=-\left(C_{\mathrm{hel}}+C_{\mathrm{nn}}+C_{\mathrm{rr}}\right) / 3$ within appropriate $M_{t \bar{t}}$ bins in order to enhance the signal. These spin correlations are equivalent to angular correlations between the leptonic decay products of the top quark:

$$
C_{\mathrm{xx}}=-9\left\langle\cos \theta_{x,+} \cos \theta_{x,-}\right\rangle, \quad D=-3\left\langle\cos \theta_{+,-}\right\rangle, \quad \cos \theta_{x, \pm}=\hat{\mathbf{x}} \cdot \hat{\boldsymbol{\ell}}_{ \pm}, \quad \cos \theta_{+,-}=\hat{\boldsymbol{\ell}}_{+} \cdot \hat{\boldsymbol{\ell}}_{-},
$$


where $\langle O\rangle=\sigma^{-1} \int d \sigma O$ denotes the expectation value of the observable $O$ and $\hat{\mathbf{x}}=\{\hat{\mathbf{k}}, \hat{\mathbf{n}}, \hat{\mathbf{r}}\}$. Here $\hat{\mathbf{k}}$ is the direction of flight of the top quark in the $t \bar{t}$ zero-momentum frame, $\hat{\boldsymbol{\ell}}_{\mp}$ is the direction of flight of the lepton $\ell_{-}$(anti-lepton $\ell_{+}$) in the anti-top (top) rest frame. The vectors $\hat{\mathbf{n}}$ and $\hat{\mathbf{r}}$ are defined as

$$
\hat{\mathbf{n}}=\frac{\hat{\mathbf{p}} \times \hat{\mathbf{k}}}{|\hat{\mathbf{p}} \times \hat{\mathbf{k}}|}, \quad \hat{\mathbf{r}}=\frac{\hat{\mathbf{p}}-y \hat{\mathbf{k}}}{|\hat{\mathbf{p}}-y \hat{\mathbf{k}}|}, \quad y=\hat{\mathbf{p}} \cdot \hat{\mathbf{k}}
$$

and $\hat{\mathbf{p}}$ is the direction of flight of the incoming proton in the laboratory frame.

In Fig. 2 we display the LO and NLO QCD results for the four different spin correlations in scenario 4 for $2 m_{t} \leq M_{t \bar{t}} \leq 400 \mathrm{GeV}$. The upper plot shows the values of the spin correlations at LO and NLO for the SM contribution and the combined SM and 2HDM contributions. The darker parts of the bars in the upper plot indicate the uncertainties due to renormalization and factorization scale variations. In the lower plot the corresponding ratios between the $2 \mathrm{HDM}$ contributions and the SM contributions are shown. The error bars indicate the change due to scale variations. It seems that the NLO results show a stronger dependence on the renormalization and factorization scales than the LO even though one would expect the opposite behavior. At LO the renormalization scale dependence cancels in the ratio. As a consequence the variation of $\mu_{0}$ does not give a reliable estimate of the uncertainty of the LO result. However, the NLO corrections affect the ratios only slightly and increase their absolute values by a few percent.

The largest effect of heavy Higgs bosons in $t \bar{t}$ production can be seen in $C_{\mathrm{rr}}$, which shows a deviation of $\sim 15 \%$ from its SM value. This is to be compared with the signal-to-background ratio of $\sim 4 \%$ for the cross section in the same $M_{t \bar{t}}$ bin. It clearly shows that spin dependent observables such as spin correlations significantly increase the sensitivity to heavy Higgs bosons.

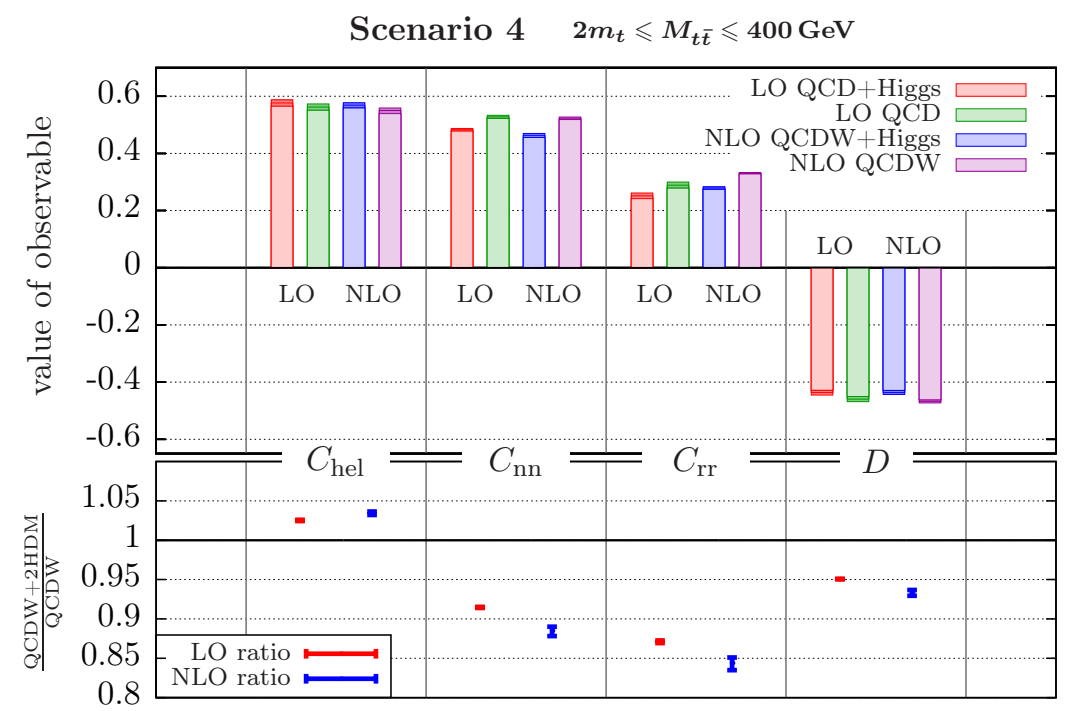

Figure 2: Spin correlations for scenario 4 at LO and NLO. Upper plot: values of the observables, lower plot: signal-to-background ratios. 


\section{Conclusion}

We calculated, within the type-II 2HDM extension of the SM, the NLO QCD corrections to the resonant production of heavy Higgs bosons and their decay into top-quark pairs at the LHC, taking into account the non-resonant SM $t \bar{t}$ continuum and its interference with the signal. We studied $C P$-conserving and $C P$-violating scenarios and investigated several observables. The interference contribution generates a peak-dip structure that leads to cancellations in inclusive observables. As a consequence the total cross sections for our 2HDM scenarios show only small signal-tobackground ratios of about 1-2\%. Differential distributions in combination with cuts on $M_{t \bar{t}}$ can give larger effects. In scenario 1 where the two resonances overlap we calculated a signal-tobackground ratio of up to $6 \%$ for the distributions of $M_{t \bar{t}}$ and the Collins-Soper angle. While this is already an increase of S/B by a factor of three, less optimistic parameter scenarios call for a higher sensitivity. We showed that this can be achieved by studying $t \bar{t}$ spin correlations. As an example we presented the results for a type-II 2HDM scenario that contains two heavy Higgs bosons with masses of $400 \mathrm{GeV}$ and $900 \mathrm{GeV}$ and Yukawa couplings to top quarks of SM strength. Within this scenario the spin correlation $C_{\mathrm{rr}}$ shows the largest signal-to-background ratio of about $15 \%$. In comparison with the signal-to-background ratio of $\sim 4 \%$ of the total cross section evaluated in the same $M_{t \bar{t}}$ bin as $C_{\mathrm{rr}}$ this amounts to an increase of about a factor of four in sensitivity. In this analysis we have shown that the challenges of searching for heavy Higgs resonances in the $t \bar{t}$ decay channel can partly be overcome by a combination of top-spin independent and spin-dependent observables evaluated in suitable $M_{t \bar{t}}$ windows.

\section{References}

[1] CMS collaboration, V. Khachatryan et al., Precise determination of the mass of the Higgs boson and tests of compatibility of its couplings with the standard model predictions using proton collisions at 7 and 8 TeV, Eur. Phys. J. C75 (2015) 212, [1412.8662].

[2] ATLAS collaboration, G. Aad et al., Measurements of the Higgs boson production and decay rates and coupling strengths using pp collision data at $\sqrt{s}=7$ and $8 \mathrm{TeV}$ in the ATLAS experiment, Eur. Phys. J. C76 (2016) 6, [1507.04548].

[3] ATLAS collaboration, G. Aad et al., A search for tit resonances using lepton-plus-jets events in proton-proton collisions at $\sqrt{s}=8 \mathrm{TeV}$ with the ATLAS detector, JHEP 08 (2015) 148, [1505.07018].

[4] ATLAS collaboration, Search for heavy Higgs bosons A/H decaying to a top-quark pair in pp collisions at $\sqrt{s}=8 \mathrm{TeV}$ with the ATLAS detector, ATLAS-CONF-2016-073.

[5] CMS collaboration, S. Chatrchyan et al., Searches for new physics using the $t \bar{t}$ invariant mass distribution in pp collisions at $\sqrt{s}=8 \mathrm{TeV}$, Phys. Rev. Lett. 111 (2013) 211804, [1309. 2030].

[6] CMS collaboration, V. Khachatryan et al., Search for resonant $t \bar{t}$ production in proton-proton collisions at $\sqrt{s}=8 \mathrm{TeV}$, Phys. Rev. D93 (2016) 012001, [1506.03062].

[7] W. Bernreuther, P. Galler, C. Mellein, Z. G. Si and P. Uwer, Production of heavy Higgs bosons and decay into top quarks at the LHC, Phys. Rev. D93 (2016) 034032, [1511.05584].

[8] B. Hespel, F. Maltoni and E. Vryonidou, Signal background interference effects in heavy scalar production and decay to a top-anti-top pair, 1606.04149.

[9] G. C. Branco, P. M. Ferreira, L. Lavoura, M. N. Rebelo, M. Sher and J. P. Silva, Theory and phenomenology of two-Higgs-doublet models, Phys. Rept. 516 (2012) 1-102, [1106.0034].

[10] W. Bernreuther, P. Galler, Z. G. Si and P. Uwer, to be published, . 